\title{
Loewner Interpolation in Matrix Hardy Classes
}

\author{
D. Alpay and J. Leblond
}

\begin{abstract}
We study a Loewner type interpolation problem in the framework of matrix-valued $\mathbf{H}_{2}$ functions. We establish a necessary and sufficient condition for a matrix-valued function given on an arc of circle to be the trace of such an $\mathbf{H}_{2}$ function subject to some constraint.
\end{abstract}

Keywords: Loewner interpolation, reproducing kernel spaces, Hardy spaces

AMS subject classification: Primary 47A57, secondary 30E05

\section{Introduction and motivations}

The problem of recapturing an analytic function from its values on a subset of the boundary originates with the work of K. Loewner in his 1934 paper [11], and has been since then considered by a number of authors; we refer to the monographs [1] and [13] for discussion and references. The present work, which is a continuation of [3], is in particular related to the work of M. G. Krein and P. Ja. Nudelman [10], of D. Patil [12] and of M. Rosenblum and J. Rovnyak [13]. We consider an interpolation problem of Loewner type for matrix-valued functions. Both the motivation for the problem and the method to solve it stem mainly from the theory of reproducing kernel Hilbert spaces. However, part of our interest for this question arise from system theoretic problems related to identification of stable infinite dimensional systems from frequency data. These aspects will be detailed below.

We consider the following problem:

Problem (P): Given a subset $I$ of the unit circle $\mathbf{T}$, measurable and of positive measure, and given a $\boldsymbol{C}^{p \times m}$-valued function $f$ with components in $\mathbf{L}_{2}(I)$, find necessary and sufficient conditions on $f$ for a $\boldsymbol{C}^{p \times m}$-valued function $h$ to exist with the following properties:

1. The entries of $h$ are in the Hardy space $\mathbf{H}_{2}$ of the unit disk.

2. It holds that

$$
\frac{1}{2 \pi} \int_{0}^{2 \pi} h\left(e^{i t}\right)^{*} h\left(e^{i t}\right) d t \leq I_{m} .
$$

D. Alpay: Ben Gurion Univ. of the Negev, Dep. Math., POB 653, Beer Sheva 84105, Israel J. Leblond: INRIA, B.P. 93, 06902 Sophia-Antipolis Cedex, France 
3. Almost everywhere on $I$, we have

$$
f\left(e^{i t}\right)=h\left(e^{i t}\right)
$$

Problem (P) is solved by the following result.

Theorem 1.1: Given $f$ and $I$ as above, there exists a function $h$ satisfying conditions (1.1) and (1.2) if and only if

$$
\sum_{n=0}^{\infty}\left\|\int_{I} \phi\left(e^{i t}\right) e^{i n t} d t\right\|^{2} \geq\left\|\int_{I} f\left(e^{i s}\right)^{*} \phi\left(e^{i s}\right) d s\right\|^{2}
$$

for every $\boldsymbol{C}^{p}$-valued function $\phi$ continuous on $I$, where $\|\cdot\|$ denotes the Euclidean norm.

Theorem 1.1 generalizes to the case of $\boldsymbol{C}^{p \times m}$-valued functions - the results of the papers [3] and [13]. Another characterisation of traces of functions in Hardy classes, together with an approximation procedure, was first given in [10], where the following result is proved.

For a given function $f \in L_{2}(-1,1)$ to have an extension to the Hardy space of the open upper half-plane, it is necessary and sufficient that $\exp (\pi \cdot) \phi_{f} \in L_{2}(\mathbb{R})$, where

$$
\phi_{f}(\lambda)=\int_{-\infty}^{+\infty} \frac{f\left(\tan \frac{x}{2}\right) \exp (-i \lambda x)}{\cosh \frac{x}{2}} d x
$$

In the following, $\mathbf{H}_{2}^{p \times m}$ stands for the space of $\boldsymbol{C}^{p \times m}$-valued functions with $\mathbf{H}_{2}$ entries.

An interpretation of problem (P) and Theorem 1.1 may be provided as follows in terms of system theory. Let

$$
h(z)=\sum_{k \geq 0} h_{k} z^{k} \quad \text { with } h_{k} \in \boldsymbol{C}^{p \times m}
$$

be the power series expansion of $h$ at the origin, and define a discrete time causal system by

$$
y_{k}=\sum_{l=0}^{k} h_{l} u_{k-l} \quad(k=0,1, \ldots)
$$

where $\left(u_{l}\right)_{l \geq 0}$ is an input sequence in $\boldsymbol{C}^{m}$ and $\left(y_{k}\right)_{k \geq 0}$ is the associated output sequence with values in $\boldsymbol{C}^{\boldsymbol{p}}$. In the present framework, $h$ will be referred to as the transfer function of the system (S). Note that, classically; the transfer function of (S) is $h\left(\frac{1}{z}\right)$ rather than $h(z)$. A precise interpretation of condition (1.1) in terms of $l_{1} / l_{2}$ stability of the system $(S)$ is established later which goes as follows (see Theorem 5.1 and also [6]). The transfer function $h$ belongs to $\mathbf{H}_{2}^{p \times m}$ if and only if, for each $l_{1}^{m}$ input sequence, the output sequence of (S) belongs to $l_{2}^{p}$; in this case, $h$ satisfies (1.1) if and only if $\left\|\left(y_{k}\right)\right\|_{l_{2}^{m}} \leq\left\|\left(u_{l}\right)\right\|_{l_{1}^{p}}$ for each $l_{1}^{m}$ input sequence $\left(u_{l}\right)$. 
There are many practical situations where a transfer function $h$ is given only by means of some of its (possibly noisy) values at some frequencies of the bandwidth of the system, obtained by harmonic identification (see [7]). This corresponds to the knowledge of the non-tangential limits of $h$ at some points contained in an arc of circle I. An interpolant $f \in \mathbf{L}_{2}(I)^{p \times m}$ is easily constructed from these values. When the system under study is $l_{1} / l_{2}$ stable and dissipative, its identification will be performed by finding a function $h \in \mathbf{H}_{2}^{p \times m}$ satisfying (1.1) such that the restriction $h_{\left.\right|_{I}}$ is as closed as possible to $f$ in $\mathbf{L}_{2}(I)^{p \times m}$. Theorem 1.1 provides an explicit way to verify if $f$ is already the trace on $I$ of an $\mathbf{H}_{2}^{p \times m}$ function. When this holds, $h$ will be an interpolant of $f$ on $I$ while, elsewhere, $h$ is to be viewed as an approximant to $f$ in $L_{2}(I)^{p \times m}$. In any case, an explicit constructive scheme for $h$ goes as follows (see [4] and [7] for details).

Let $P_{\mathbf{H}_{2}}$ denote the orthogonal projection from $\mathrm{L}_{2}(\mathrm{~T})$ onto $\mathbf{H}_{2} ; \chi_{I}$ the characteristic function of the subset $I \subset \mathbf{T}$, and $\Phi$ the Toeplitz operator on $\mathbf{H}_{2}$ with symbol $\chi_{\mathbf{T} \backslash I}$. For any $f \in \mathbf{L}_{2}(I)$, the family of $\mathbf{H}_{2}^{p \times m}$ functions defined by

$$
h_{\lambda}=\left[(1+\lambda \Phi)^{-i} P_{\mathbf{H}_{2}}\left(\chi_{I} f_{k, l}\right)\right]_{\substack{k=1, \ldots, p \\ l=1, \ldots, m}}
$$

for $\lambda>-1$ converges to $f$ in $\mathbf{L}_{2}(I)^{p \times m}$ as $\lambda \rightarrow-1$. This has been established in [4] in the scalar case $p=m=1$ and may be easily generalized to the present multivariable problem. Now, it follows from [12] and a straightforward computation that whenever $f$ is the trace on $I$ of an $\mathbf{H}_{2}^{p \times m}$ function $h$, then $\left(h_{\lambda}\right)$ converges to $h$ in $\mathbf{H}_{2}^{p \times m}$. Observe that this provides another way to characterize traces on $I$ of $\mathbf{H}_{2}^{p \times m}$ functions. Indeed, $\left\|h_{\lambda}\right\|_{L_{2}(T)}$ remains bounded as $\lambda \rightarrow-1$ if and only if $f$ is the restriction to $I$ of an $\mathbf{H}_{2}^{p \times m}$ function (see also [4] and [7]).

Furthermore, it is proved in [2] (and [15] for the scalar case) that a function $h \in$ $\mathbf{H}_{2}^{p \times m}$ satisfies (1.1) if and only if

$$
h(z)=S_{1}(z)\left(I_{m}-z S_{2}(z)\right)^{-1}
$$

where $S_{1}$ and $S_{2}$ are analytic in $\mathbb{D}, \boldsymbol{C}^{p \times m}$ - and $\boldsymbol{C}^{m \times m}$-valued, respectively, and where $S=\left(\begin{array}{c}S_{1} \\ S_{2}\end{array}\right)$ is a Schur function, that is

$$
S_{1}(z)^{*} S_{1}(z)+S_{2}(z)^{*} S_{2}(z) \leq I_{m}
$$

in $\mathbb{D}$. Assume that

$$
S_{i}(z)=\sum_{k \geq 0} \sigma_{k}^{(i)} z^{k} \quad(i=1,2) .
$$

Then $h$ is the transfer function of the following "hereditary" system:

$$
\begin{aligned}
& x_{0}=u_{0} \\
& x_{k}=u_{k}+\sum_{l=0}^{k-1} \sigma_{l}^{(2)} x_{k-l-1} \quad(k \geq 1) \\
& y_{k}=\sum_{l=0}^{k} \sigma_{l}^{(1)} x_{k-l} \quad(k \geq 0) .
\end{aligned}
$$

Such a system may be viewed in particular as the discretized analogue of an integrodifferential (continuous) system: 


\section{Positivity and $H_{2}$ functions}

Let $K(z, w)$ be a $\boldsymbol{C}^{m \times m}$-valued function defined for $z, w$ in some set $\Omega$. It is called nonnegative if it is Hermitian, i.e. $K(z, w)^{*}=K(w, z)$, and if for every choice of integer $r$, every choice of points $\omega_{1}, \ldots, \omega_{r} \in \Omega$ and vectors $\xi_{1}, \ldots, \xi_{r} \in \boldsymbol{C}^{m}$, the $(r \times r)$-Hermitian matrix with $l k$ entry $\xi_{l}^{*} K\left(\omega_{l}, \omega_{k}\right) \xi_{k}$ is non-negative. Associated to such a non-negative function is a unique reproducing kernel Hilbert space $H(K)$ (of $\boldsymbol{C}^{\mathbf{m}}$-valued functions) with reproducing kernel $K(z, w)$, i.e. with the following two properties:

1. For every choice of $\omega \in \Omega$ and $\xi \in \boldsymbol{C}^{m}$, the function $z \mapsto K(z, \omega) \xi$ belongs to $H\left(K^{\prime}\right)$.

2. For all $f \in H(K)$ and $\omega, \xi$ as above,

$$
\langle f, K(\cdot, \omega) \xi\rangle_{H(K)}=\xi^{*} f(\omega)
$$

where $\langle\cdot, \cdot\rangle_{H(K)}$ denotes the inner product in $H(K)$.

There is a one-to-one correspondence between reproducing kernel Hilbert spaces and non-negative functions (see $[5,14,16]$ for this and for the general theory of such spaces). We will refer to non-negative functions also as kernel functions.

An example which will be of importance in the present framework is the case of functions of the Hardy space $\mathbf{H}_{2}$ of the disk (see, e.g., $[8,9,14]$ for the definition of these spaces). The space $\mathbf{H}_{2}$ has reproducing kernel $\frac{1}{1-z w^{*}}$, as it may be deduced from Cauchy's formula. The following theorem gives a characterization of functions of $\mathbf{H}_{2}$ of norm less than 1; for a proof of it, as well as for a proof of its matrix-valued counterpart (Theorem 2.2), we refer to [2].

Theorem 2.1: Let $h$ be analytic in the unit disk $I D$. Then, $h$ is in $\mathbf{H}_{2}$ and $\|h\|_{\mathbf{H}_{2}} \leq$ 1 if and only if the function

$$
K_{h}(z, w)=\frac{1}{1-z w^{*}}-h(z) h(w)^{*}
$$

is non-negative in $\mathbb{D}$.

When one considers elements of the space $\mathbf{H}_{2}^{p \times m}$ of $\boldsymbol{C}^{p \times m}$-valued functions with entries in $\mathbf{H}_{2}$, the preceding result has to be suitably modified. Let us first define on $\mathbf{H}_{2}^{p \times m}$ a matrix-valued Hermitian form by

$$
[h, h]=\frac{1}{2 \pi} \int_{0}^{2 \pi} h\left(e^{i t}\right)^{*} h\left(e^{i t}\right) d t .
$$

The space $\mathbf{H}_{2}^{p \times m}$ is a Hilbert space when endowed with the norm $\sqrt{\operatorname{Tr}[h, h]}$.

Theorem 2.2: Let $h$ be a $C^{p \times m}$-valued function analytic in $\mathbb{D}$. Then, it belongs to $\mathbf{H}_{2}^{p \times m}$ and $[h, h] \leq I_{m}$ if and only if the function

$$
K_{h}(z, w)=\frac{I_{p}}{1-z w^{*}}-h(z) h(w)^{*}
$$


is non-negative in $D$.

It is of interest to note that one could suppose only $h$ defined on $D$, with no other hypothesis. The non-negativity of the function (2.3) already forces the function $h$ to be analytic (and in fact, to belong to $\mathbf{H}_{2}^{p \times m}$ ).

Comparison of the two theorems suggests that, for the $\mathbf{H}_{2}^{p \times m}$ case, the natural analogue of the condition $\|h\|_{\mathrm{H}_{2}} \leq 1$ is $[h, h] \leq I_{m}$, and not $\operatorname{Tr}[h, h] \leq 1$ (at least from the point of view of reproducing kernel spaces). Indeed, if $[h, h] \leq I_{m}$, then $\operatorname{Tr}[h, h] \leq m$. Conversely, if $\operatorname{Tr}[h, h] \leq k$ for some $k>0$, then $[h, h] \leq k I_{m}$, but when $p$ and $m$ are both greater than 1 , there is no pair $\left(k_{1}, k_{2}\right)$ of strictly positive numbers such that the conditions $\operatorname{Tr}[h, h] \leq k_{1}$ and $[h, h] \leq k_{2} I_{m}$ are equivalent. We show this

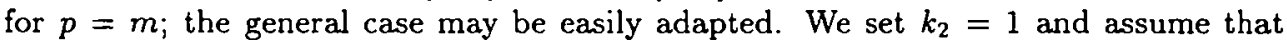
there is $k_{1}$ with the above property. Let $h^{\sharp}(z)=h\left(z^{*}\right)^{*}$. Since $\operatorname{Tr}[h, h]=\operatorname{Tr}\left[h^{\sharp}, h^{\sharp}\right]$, the condition (1.1) would then be equivalent to the condition

$$
\frac{1}{2 \pi} \int_{0}^{2 \pi} h\left(e^{i t}\right) h\left(e^{i t}\right)^{*} d t \leq I_{p}
$$

To check that this is false take for instance

$$
h(z)=\left(\begin{array}{cccc}
1 & 0 & 0 & \cdots \\
0 & 0 & 0 & \cdots \\
0 & \cdots & & \\
\vdots & & &
\end{array}\right)+z\left(\begin{array}{cccc}
0 & 1 & 0 & \cdots \\
0 & 0 & 0 & \cdots \\
0 & \cdots & & \\
\vdots & & &
\end{array}\right)
$$

Proposition 2.3: Let $0<r<1$ and let $C_{r}=\{z \in \boldsymbol{C}:|z|=r\}$. Let $K(z, w)$ be a $C^{m \times m}$-valued function which is non-negative on $C_{r}$. Suppose moreover that $K(z, w)$ is jointly continuous in $z, w \in C_{r}$. Then, for every continuous function $\phi: \mathbf{T} \rightarrow \boldsymbol{C}^{\mathbf{m}}$,

$$
\int_{0}^{2 \pi 2 \pi} \int_{0} \dot{\phi}\left(e^{i t}\right)^{*} K\left(r e^{i t}, r e^{i s}\right) \phi\left(e^{i s}\right) d t d s \geq 0 .
$$

Proof: The integral (2.5) can be written as the limit of finite sums of the form

$$
\sum_{l, k} \phi\left(e^{i t_{l}}\right)^{*} K\left(r e^{i t_{1}}, r e^{i t_{k}}\right) \phi\left(e^{i t_{k}}\right)
$$

where the $t_{k} \in[0,2 \pi]$. These sums are non-negative, thanks to the non-negativity of $K(z, w)$, from which the inequality (2.5) follows 


\section{Proof of Theorem 1.1: Necessity of the condition (1.3)}

Let us assume that a function $h \in \mathbf{H}_{2}^{p \times m}$ exists satisfying (1.1) and (1.2). From Theorem 2.2, the kernel (2.3) is non-negative. Therefore, by Proposition 2.3, for every continuous function $\phi: \mathbf{T} \rightarrow \boldsymbol{C}^{p}$ and every $r$ such that $0<r<1$,

$$
\int_{0}^{2 \pi 2 \pi} \int_{0}\left(\frac{\phi\left(e^{i t}\right)^{*} \phi\left(e^{i s}\right)}{1-r^{2} e^{i t} e^{-i s}}-\phi\left(e^{i t}\right)^{*} h\left(r e^{i t}\right) h\left(r e^{i s}\right)^{*} \phi\left(e^{i s}\right)\right) d t d s \geq 0
$$

Taking $\phi$ continuous on $\mathbf{T}$ and vanishing outside $I$, we obtain (1.3) from (3.1). Indeed, for such $\phi$,

$$
\sum_{n=0}^{\infty}\left\|\int_{I} e^{i n s} \phi\left(e^{i s}\right) d s\right\|^{2}<\infty
$$

and so,

$$
\int_{0}^{2 \pi 2 \pi} \int_{0}^{\phi\left(e^{i t}\right)^{*} \phi\left(e^{i s}\right)} \frac{r^{2} e^{i t} e^{-i s}}{1-\infty} d t s=\sum_{n=0}^{\infty n} r^{2 n}\left\|\int_{l} \phi\left(e^{i s}\right) e^{i n s} d s\right\|^{2}
$$

and

$$
\lim _{r \rightarrow 1} \int_{0}^{2 \pi 2 \pi} \int_{0}^{\phi\left(e^{i t}\right)^{*} \phi\left(e^{i s}\right)} \frac{r^{2} e^{i t} e^{-i s}}{1-\infty} d s=\sum_{n=0}^{\infty}\left\|\int_{I} \phi\left(e^{i s}\right) e^{i n s} d s\right\|^{2} .
$$

On the other hand,

$$
\lim _{r \rightarrow 1} \int_{I} \phi\left(e^{i t}\right)^{*} h\left(r e^{i t}\right) d t=\int_{I} \phi\left(e^{i t}\right)^{*} h\left(e^{i t}\right) d t=\int_{I} \phi\left(e^{i t}\right)^{*} f\left(e^{i t}\right) d t
$$

where the first equality follows from the fact that, when $h \in \mathbf{H}_{2}^{p \times m}$ and $0<r<1$, the functions $z \mapsto h(r z)$ tend to $h$ in the $\mathbf{H}_{2}^{p \times m}$ norm and where we use (1.2) to go from the first line to the second one. Equality (1.3) follows now from (3.1).

\section{Proof of Theorem 1.1: The sufficiency}

We follow the arguments of [3], suitably adapted to the vector case. We first introduce two scalar functions $K_{0}(\phi, \psi)$ and $K_{1}(\phi, \psi)$, which are non-negative on the set $\Omega$ of $\boldsymbol{C}^{p}$-valued functions continuous on $I$, by

$$
\begin{aligned}
& K_{0}(\phi, \psi)=\sum_{n=0}^{\infty}\left(\int_{I} \psi\left(e^{i t}\right)^{*} e^{i n t} d t\right)\left(\int_{I} \phi\left(e^{i s}\right) e^{-i n s} d s\right) \\
& K_{1}(\phi, \psi)=\left(\int_{I} \psi\left(e^{i t}\right)^{*} f\left(e^{i t}\right) d t\right)\left(\int_{I} f\left(e^{i s}\right)^{*} \phi\left(e^{i s}\right) d s\right) .
\end{aligned}
$$


The associated reproducing kernel Hilbert spaces are readily seen to be characterized as

$$
H\left(K_{0}\right)=\left\{F: \phi \mapsto \int_{I} x\left(e^{i s}\right)^{*} \phi\left(e^{i s}\right) d s \mid x \in \mathbf{H}_{2}^{1 \times p}\right\}
$$

with norm

$$
\|F\|_{H\left(K_{0}\right)}=\|x\|_{\mathbf{H}_{2}^{1 \times p}}
$$

and

$$
H\left(K_{1}\right)=\left\{F: \phi \mapsto \int_{I} \eta f\left(e^{i s}\right)^{*} \phi\left(e^{i s}\right) d s \mid \eta \in \boldsymbol{C}^{1 \times m}\right\}
$$

with norm

$$
\|F\|_{H\left(K_{1}\right)}=\|(I-\pi) \eta\|_{C^{m}}
$$

respectively, $\pi$ being the orthogonal projection on the set $\left\{\eta \in \boldsymbol{C}^{1 \times m}: \eta f\left(e^{i s}\right)^{*} \equiv 0\right\}$. Let us assume that (1.3) holds. We rewrite it as

$$
\begin{aligned}
\sum_{n=0}^{\infty}\left(\int_{I} \phi\left(e^{i t}\right)^{*} e^{i n t} d t\right) & \left(\int_{I} \phi\left(e^{i s}\right) e^{-i n s} d s\right) \\
& \geq\left(\int_{I} \phi\left(e^{i t}\right)^{*} f\left(e^{i t}\right) d t\right)\left(\int_{I} f\left(e^{i s}\right)^{*} \phi\left(e^{i s}\right) d s\right) .
\end{aligned}
$$

Since $K_{0}$ and $K_{1}$ are linear in $\phi$ and $\psi^{*}$, inequality (4.7) expresses the fact that the kernel $K_{0}-K_{1}$ is non-negative on $\Omega$. Therefore, the space $H\left(K_{1}\right)$ is contractively included in the space $H\left(K_{0}\right)$ (see, e.g., [5: Prop. 12] and [16]). Thus, for every $\eta \in C^{1 \times m}$, there exists $x_{\eta} \in \mathbf{H}_{2}^{1 \times p}$ such that, for every $\phi \in \Omega$,

$$
\int_{I} \eta f\left(e^{i s}\right)^{*} \phi\left(e^{i s}\right) d s=\int_{I} x_{\eta}\left(e^{i s}\right)^{*} \phi\left(e^{i s}\right) d s
$$

and

$$
\left\|x_{\eta}\right\|_{\mathbf{H}_{2}^{1 \times p}} \leq\|(I-\pi) \eta\|_{C^{1 \times p}} \leq\|\eta\|_{C^{1 \times p}} .
$$

The correspondence $\eta \mapsto x_{\eta}^{*}$ is clearly linear and so we can write $x_{\eta}^{*}=\eta h^{*}$ where $h \in$ $\mathrm{H}_{2}^{p \times m}$. By density of the functions $\phi$ in $L_{2}^{m}(I)$, equality (4.8) leads to $\eta f\left(e^{i s}\right)^{*}=\eta h\left(e^{i s}\right)$ almost everywhere on $I$. Furthermore, (4.9) leads to

$$
\frac{1}{2 \pi} \int_{0}^{2 \pi} \eta h\left(e^{i s}\right)^{*} h\left(e^{i s}\right) \eta^{*} \leq \eta \eta^{*}
$$

i.e. $[h, h] \leq I_{m}$, which ends the proof. 


\section{5. $l_{1} / l_{2}$ stability}

In this section, we establish precisely the correspondence already mentioned in the introduction between functions satisfying properties 1,2 , and 3 stated in problem (P) and transfer functions of some stable discrete time input-output systems $(\mathrm{S})$.

We denote by $l_{1}^{m}$ the space of sequences $\left(u_{k}\right)_{k \geq 0}$ of $C^{m}$ vectors, indexed by $\mathbb{N}$, and such that

$$
\|u\|_{l_{1}^{m}}=\sum_{k=0}^{\infty} \sqrt{u_{k}^{*} u_{k}}<\infty
$$

Similarly, $l_{2}^{p}$ denotes the space of sequences $\left(y_{k}\right)_{k \geq 0}$ of $\boldsymbol{C}^{p}$ vectors indexed by $\mathbb{N}$ and such that

$$
\|y\|_{l_{2}^{p}}=\sqrt{\sum_{k=0}^{\infty} y_{k}^{*} y_{k}}<\infty .
$$

We denote by $\left(l_{1}^{m}\right)_{0}$ and $\left(l_{2}^{p}\right)_{0}$ the subspaces of finite sequences of these spaces.

Theorem 5.1: Let $T \vdots\left(l_{1}^{m}\right)_{0} \rightarrow\left(l_{2}^{p}\right)$ be the operator defined by (S). Then, $T$ extends to a contraction $l_{1}^{m} \rightarrow l_{2}^{p}$ if and only if $h$ belongs to $\mathbf{H}_{2}^{p \times m}$ and satisfies the condition (1.1).

Proof: We first suppose that $T$ defines a contraction from $l_{1}^{m}$ into $l_{2}^{p}$. Let $\xi \in \boldsymbol{C}^{m}$ and let $\left(u_{k}\right)$ be the sequence defined by $u_{0}=\xi$ and $u_{k}=0$ for $k \geq 1$. Then $\left(y_{k}\right)=$ $\left(h_{k} \xi\right)(k \geq 0)$ is the output sequence. Since $\|T\| \leq 1$,

$$
\left\|\left(y_{k}\right)\right\|_{i_{2}^{p}}^{2}=\|h \xi\|_{\mathbf{H}_{2}^{p}}^{2} \leq \sum_{k \geq 0} \xi^{*} h_{k}^{*} h_{k} \xi \leq \xi^{*} \xi=\|\xi\|_{C^{m}}^{2}=\left\|\left(u_{k}\right)\right\|_{1}^{2}
$$

which is equivalent to $(1.1)$ as $[h, h]=\sum_{k \geq 0} h_{k}^{*} h_{k}$.

Conversely, let us assume that the sequence $\left(h_{k}\right)_{k \geq 1}$ satisfies (5.1), i.e. $h \in \mathbf{H}_{2}^{p \times m}$ and $[h, h] \leq I_{m}$. Let now $\left(u_{k}\right)_{k=0, \ldots, N}$ be a finite sequence and $u(z)=\sum_{i=0}^{N} u_{i} z^{i}$. Then,

$$
\begin{aligned}
\left\|\left(y_{k}\right)\right\|_{l_{2}^{p}} & =\|h u\|_{\mathbf{H}_{2}^{p}} \\
& =\left\|\sum_{i=0}^{N} h(z) u_{i} z^{i}\right\|_{\mathbf{H}_{2}^{p}} \leq \sum_{i=0}^{N}\left\|h(z) u_{i} z^{i}\right\|_{\mathbf{H}_{2}^{p}} \\
& =\sum_{i=0}^{N}\left\|h(z) u_{i}\right\|_{\mathbf{H}_{2}^{p}} \leq \sum_{i=0}^{N}\left\|u_{i}\right\|_{C^{m}} \\
& =\left\|\left(u_{i}\right)\right\|_{l_{1}^{m}} .
\end{aligned}
$$

This concludes the proof since finite sequences are dense in $l_{1}^{m}$ 


\section{References}

[1] Aizenberg, L.: Carleman's Formulas in Complex Analysis (Mathematics and its Applications: Vol. 244). Dordrecht: Kluwer Acad. Publ. 1993.

[2] Alpay, D., Bolotnikov, V. and Y. Peretz: The Nevanlinna-Pick $\mathbf{H}_{2}$ interpolation problem. Trans. Amer. Math. Soc. (to appear).

[3] Alpay, D. and J. Leblond: Traces of Hardy functions and reproducing kernel Hilbert spaces (submitted/short version to appear in Proc. MTNS 1993).

[4] Alpay, D., Baratchart, L. and J. Leblond: Some extremal problems linked with identification from partial frequency data. Lect. Notes Control Inf. Sci. 185 (1992), 563 573.

[5] Aronszajn, N.: Theory of reproducing kernels. Trans. Amer. Math. Soc. 68 (1950), 337 $-404$.

[6] Baratchart, L.: Sur l'approximation $L^{2}$ pour les systèmes dynamiques linéaires. Thèse d' Etat. Université de Nice 1987.

[7] Baratchart, L., Leblond, J. and J. Partington.: Hardy approzimation to $L^{p}$ functions on subsets of the circle (submitted.)

[8] Garnett, J. B.: Bounded Analytic Functions. New York - London: Academic Press 1981.

[9] Hoffman, K.: Banach Spaces of Analytic Functions. Englewood Cliffs (N. J.): Prentice Hall 1962.

[10] Krein, M. G. and P. Ja. Nudelman: Approzimation of $L_{2}\left(\omega_{1}, \omega_{2}\right)$ functions by minimumenergy transfer functions of linear systems (in Russian). Problemy Peredaci Informatsii 11 (1975)2, 37 - 60. Engl. transl. in: Problems Intern. Transm. 11 (1976), 124 - 142.

[11] Loewner, K.: Über monotone Matrixfunktionen. Math. Z. 38 (1934), 177 - 216.

[12] Patil, D.: Representation of $H^{p}$-functions. Bull. Amer. Math. Soc. 78 (1972), 617 - 620.

[13] Rosenblum, M. and J. Rovnyak: Restrictions of analytic functions. Part I. Proc. Amer. Math. Soc. 51 (1975), 113 - 119.

[14] Saitoh, S.: Theory of Reproducing Kernels and its Applications (Pitman Research Notes in Mathematics: Vol. 189). Burnt Mill - Harlow - Essex: Longman \& Scientific 1988.

[15] Sarason, D.: Exposed points in $H^{1}$. Part I. Oper. Theory: Adv. Appl. 41 (1989), 485 496.

[16] Schwartz, L.: Sous espaces hilbertiens d'espaces vectoriels topologiques et noyaux associés (noyaux reproduisants). J. d'Anal. Math. 13 (1964), 115 - 256. 